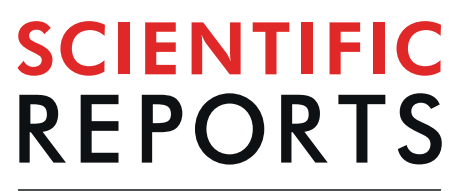

natureresearch

\title{
Analysis of neuronal ensemble activity reveals the pitfalls and shortcomings of rotation dynamics
}

\author{
Mikhail A. Lebedev ${ }^{1}$, Alexei Ossadtchi ${ }^{2}$, Nil Adell Mill $\mathbb{1 D}^{3}$, Núria Armengol Urpi ${ }^{4}$, \\ Maria R. Cervera ${ }^{3}$ \& Miguel A. L. Nicolelis ${ }^{1,5,6,7,8,9 *}$
}

Back in 2012, Churchland and his colleagues proposed that "rotational dynamics", uncovered through linear transformations of multidimensional neuronal data, represent a fundamental type of neuronal population processing in a variety of organisms, from the isolated leech central nervous system to the primate motor cortex. Here, we evaluated this claim using Churchland's own data and simple simulations of neuronal responses. We observed that rotational patterns occurred in neuronal populations when (1) there was a temporal sequence in peak firing rates exhibited by individual neurons, and (2) this sequence remained consistent across different experimental conditions. Provided that such a temporal order of peak firing rates existed, rotational patterns could be easily obtained using a rather arbitrary computer simulation of neural activity; modeling of any realistic properties of motor cortical responses was not needed. Additionally, arbitrary traces, such as Lissajous curves, could be easily obtained from Churchland's data with multiple linear regression. While these observations suggest that temporal sequences of neuronal responses could be visualized as rotations with various methods, we express doubt about Churchland et al.'s bold assessment that such rotations are related to "an unexpected yet surprisingly simple structure in the population response", which "explains many of the confusing features of individual neural responses". Instead, we argue that their approach provides little, if any, insight on the underlying neuronal mechanisms employed by neuronal ensembles to encode motor behaviors in any species.

It is well known that individual neurons in cortical motor areas transiently modulate their firing rates following a stimulus that triggers the production of a voluntary movement ${ }^{1}$. These neuronal modulations have been shown to represent various motor parameters, for example movement direction ${ }^{2}$, although the specifics of these representations are still a matter of debate ${ }^{3-5}$. With the development of multichannel recordings ${ }^{6,7}$, it has become possible to study modulations recorded in multiple cortical neurons simultaneously. This methodological advance led to many studies attempting to uncover how neuronal populations process information ${ }^{8-11}$

Among the studies on motor and premotor cortical neuronal populations, one paper by Churchland and his colleagues $^{12}$ became especially popular. In their work, Churchland et al. claimed to have discovered a unique property of cortical population activity that they called "rotational dynamics". Their analysis is based on the idea that motor cortical activity could be modeled as a dynamical system (the idea that is currently being radically expanded by new machine learning techniques ${ }^{13}$ ):

$$
\dot{X}=M_{\text {skew }} X
$$

where $X$ is a multidimensional vector representing neuronal population activity, $\dot{X}$ is its time derivative and $M_{\text {skew }}$ is the transform matrix. $M_{\text {skew }}$ has imaginary eigenvalues, meaning that $\dot{X}$ is orthogonal to $X^{14}$, which corresponds to $X$ rotating around the center of rotation at the center of coordinates (Fig. 1A). (Churchland et al. did not

\footnotetext{
${ }^{1}$ Duke Center for Neuroengineering, Duke University, Durham, NC, USA. ${ }^{2}$ Center for Bioelectric Interfaces of the Institute for Cognitive Neuroscience of the National Research University Higher School of Economics, Moscow, Russia. ${ }^{3}$ Institute of Neuroinformatics, University of Zurich and ETH Zurich, Zurich, Switzerland. ${ }^{4}$ D-MAVT ETH Zurich, Zurich, Switzerland. ${ }^{5}$ Department of Neurobiology, Duke University Medical Center, Durham, NC, USA. ${ }^{6}$ Department of Neurology, Duke University, Durham, NC, USA. ${ }^{7}$ Department of Neurosurgery, Duke University, Durham, NC, USA. ${ }^{8}$ Department of Psychology and Neuroscience, Duke University, Durham, NC, USA. ${ }^{9}$ Edmond and Lily Safra International Institute of Neurosciences of Natal, Natal, Brazil. *email: nicoleli@neuro.duke.edu
} 
A

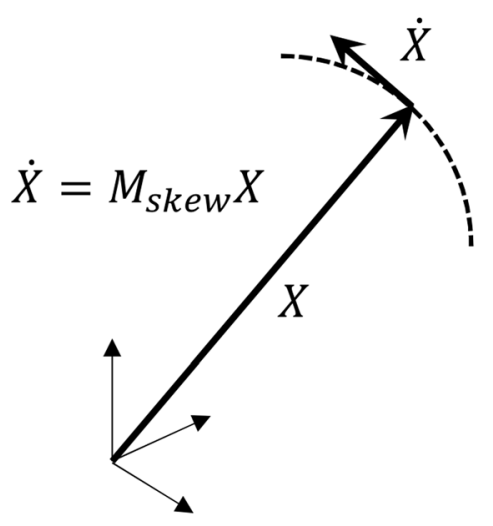

6-Dimensional
Neuronal Space
B

jPCA plane 1 (eigval ranked)

$29 \%$ of var captured

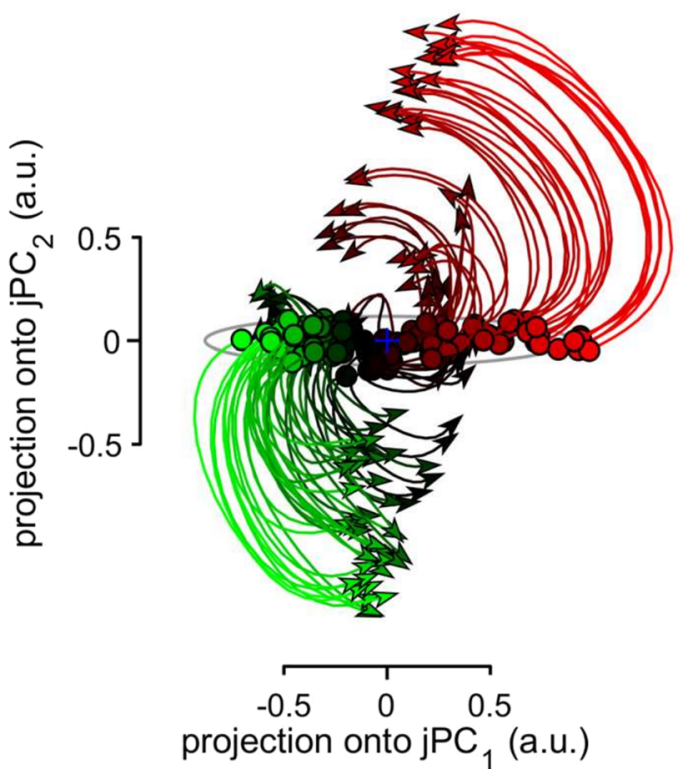

Figure 1. Rotation in a multidimensional neuronal space. (A) Schematics of "rotational dynamics", where there is an angle between the neuronal vector, $X$, and its time derivative, $\dot{X}$, meaning that $X$ is turning. In $j$ PCA proposed by Churchland et al. (2012), is $X$ is formed by the first six PCs of the population activity. (B) Application of jPCA to neuronal data. Individual curves correspond to experimental conditions, where a monkey performed armed reaching with different trajectories. The color of the curves (red, green, black) corresponds to different levels of premovement activity.

explain why rotations around a fixed center are of particular interest as opposed, for example, to curved trajectories with a varying center of rotation). In the analysis of Churchland et al., vector $X$ is produced from the activity of neuronal populations by the application of principal component analysis (PCA). The first six principal components (PCs) are selected to avoid overfitting that could take place with a larger number of dimensions. Hence, $X$ is six-dimensional. Next, a method called jPCA is applied to compute $M_{\text {skew }}$ and the corresponding eigenvectors. Churchland et al. projected PC data to the plane defined by the first two most prominent rotational components generated with jPCA and obtained the figures, where population responses rotated in the same direction for different experimental conditions (Fig. 1B).

According to the interpretation of Churchland et al., "rotational dynamics" are a fundamental feature of population activity in the motor and premotor cortical areas (but not the supplementary motor area ${ }^{15}$ ) and a proof that motor cortical populations act as a dynamical system rather than representing various motor parameters by their firing. They called the rotational effect an "orderly rotational structure, across conditions, at the population level", a "brief but strong oscillatory component, something quite unexpected for a non-periodic behavior", and "not trivial" observations that are "largely expected for a neural dynamical system that generates rhythmic output".

While this proposal has merits, we found the results of Churchland et al. difficult to comprehend because of the lack of clarity regarding the concrete neuronal patterns contributing to the rotations revealed by jPCA. For example, they suggested that "motor cortex responses reflect the evolution of a neural dynamical system, starting at an initial state set by preparatory activity [...]. If the rotations of the neural state [...] reflect straightforward dynamics, then similar rotations should be seen for all conditions. In particular, the neural state should rotate in the same direction for all conditions, even when reaches are in opposition" - a high-level description that lacks concrete details regarding activity patterns of the neurons entered in the analysis. For instance, it is unclear what "straightforward dynamics" are and why they impose the same rotational patterns on all conditions. The major obstacle to understanding the ideas expressed by Churchland and his colleagues is the absence of a clear explanation of how individual neurons and/or their populations form the rotating patterns revealed by $\mathrm{jPCA}$.

To eliminate this gap in understanding, we reanalyzed some of the data that Churchland et al. made available online. We utilized perievent time histograms (PETHs), a basic method for displaying neuronal data, to find the underlying cause for the "rotational dynamics". Next, we ran simple simulations to verify our findings. Based on our results, we found several issues with the analyses and interpretations by Churchland et al. Even though a certain temporal order in which different cortical neurons are activated during a behavioral task could be described as "rotational dynamics", we are not convinced that this observation alone could significantly "help transcend the controversy over what single neurons in motor cortex code or represent", as stated by Churchland and his colleagues. 

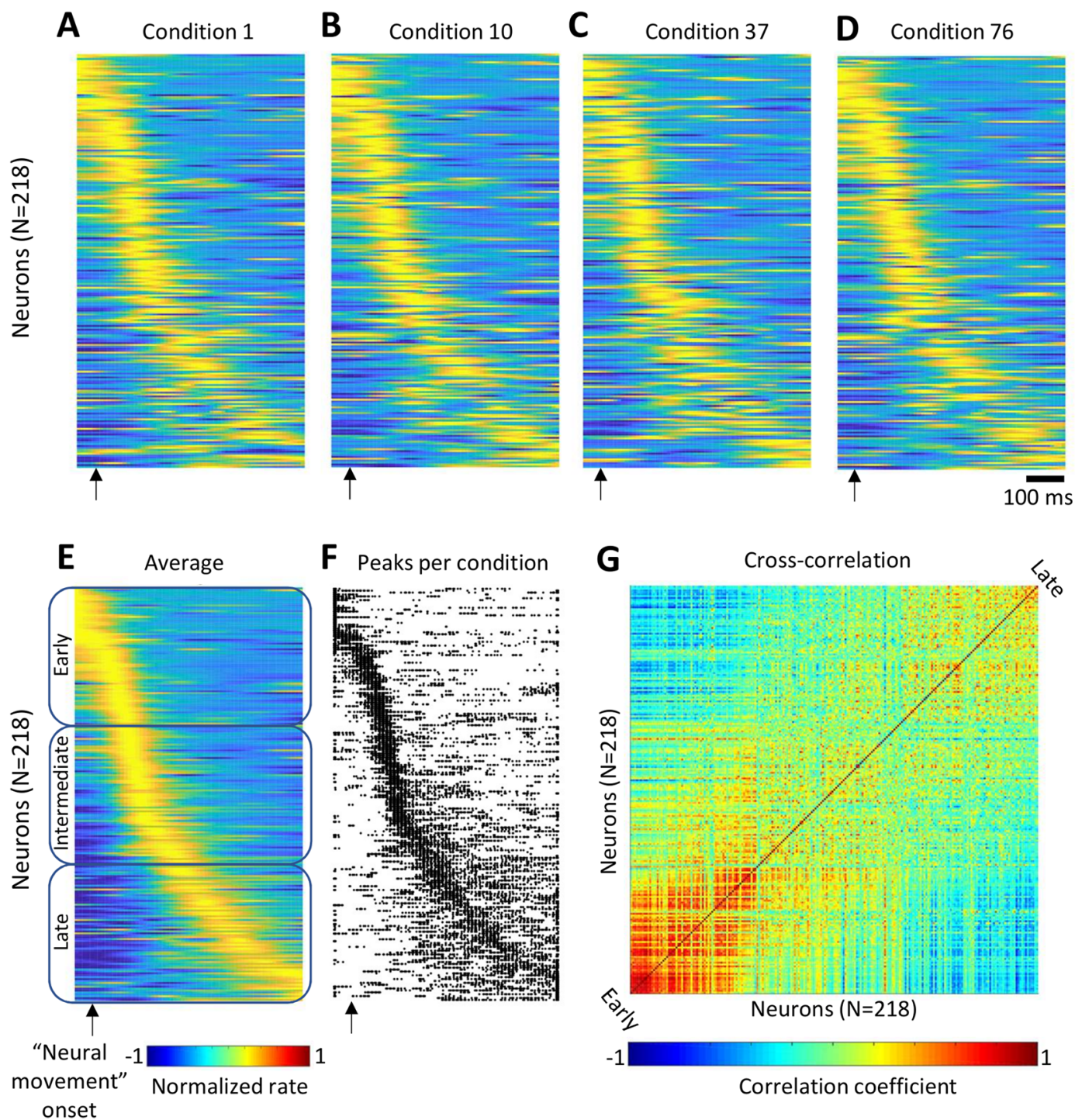

Figure 2. Time spread in peak firing rates of different neurons in the data provided by Churchland et al. (2012). (A-D) Color-coded population PETHs for four representative conditions. Horizontal lines represent PETHs for individual neurons. (E) PETHs averaged across conditions. From top to bottom: neurons are ordered by the time of peak firing rate, from the earliest activated neurons to the latest. The same order is used in (A-D,F,G). The neuronal population was dived into three subpopulations: early (neurons 1-73), intermediate (74-146), and late (147-218) responses. (F) A scatterplot showing the times of peak firing rates for different neurons and conditions. The times of peak occurrences are represented by dots. (G) Pairwise correlation between the activity patterns of different neurons.

\section{Results}

Rotation in a multidimensional neuronal space could be thought of as a process where individual neurons are activated in a certain order, which results in the neuronal vector $X$ changing orientation (Fig. 1A). Such a pattern can be also described as a phase shift between the responses of different neurons. Consider the simplest case of a population that consists of two neurons where the activity of the first neuron is a sine function of time and activity of the second neuron is a cosine. Since the phase shift between the responses of these neurons is 90 degrees, a two-dimensional plot with the firing rates of these neurons on the axes produces circular or elliptical trajectories. This type of trajectory is observed for all conditions if the phase shift between the neurons persists.

Following this logic, we hypothesized that the data of Churchland et al. contained phase shifts between the responses of different neurons, which remained consistent across conditions. To test this hypothesis, we analyzed their data using the traditional PETH method. The dataset included PETHs of 218 neurons calculated for 108 experimental conditions (i.e., one smoothed PETH per condition; single-trial data were unavailable). Each condition corresponded to a monkey performing a reaching movement with a straight or convoluted trajectory. We simply stacked these PETHs to produce population color plots for different conditions (Fig. 2A-D). Additionally, we averaged the PETHs across all conditions to obtain average responses for each neuron (Fig. 2E). For the average PETHs, we calculated peak values and reordered the neurons according to the value of the time when each 
A

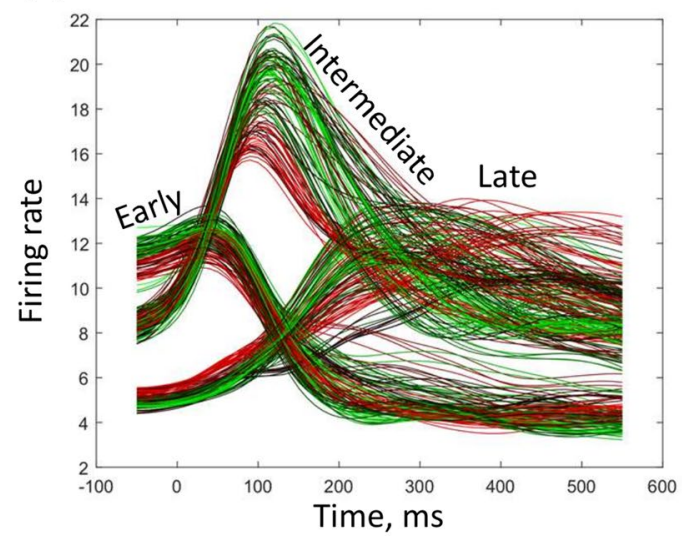

B

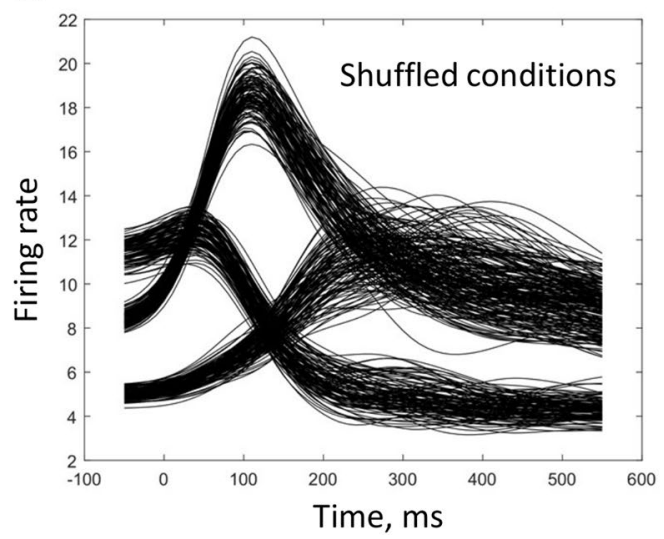

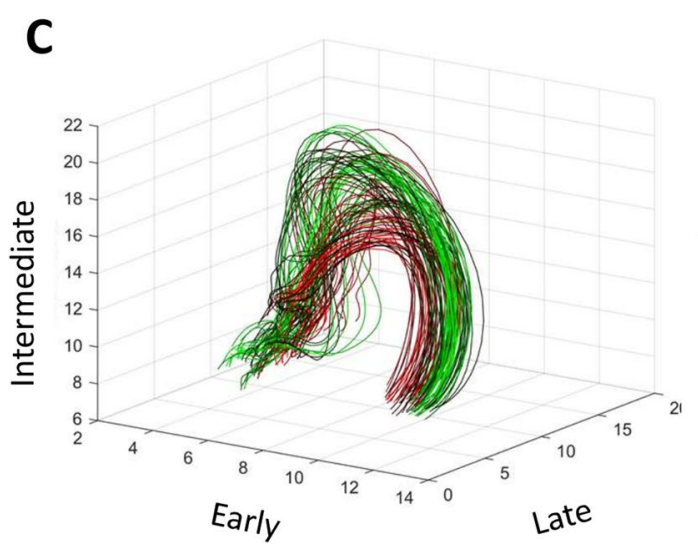

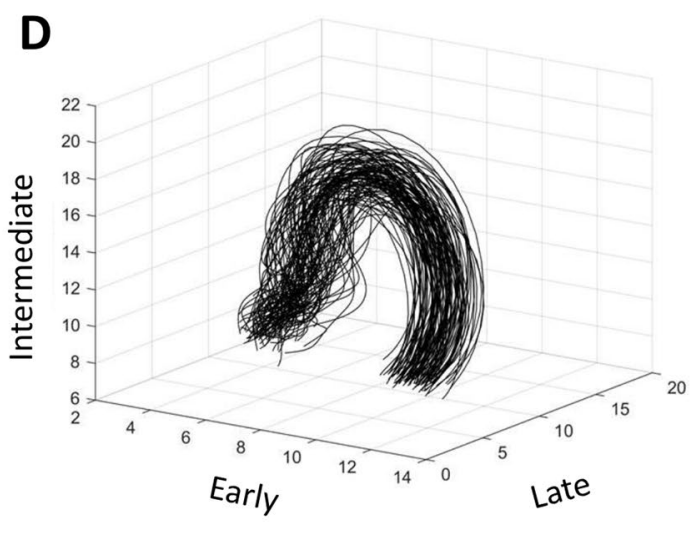

Figure 3. "Rotational dynamics" revealed by splitting neuronal population into the early, intermediate and late activated subpopulations. (A) Average PETHs for the three subpopulations, for different experimental conditions. The composition of the subpopulations is shown in Fig. 2E. Color coding of the traces is the same as in Fig. 1B. (B) Average PETHs for the shuffled-conditions data. (C,D) "Rotational dynamics" shown as threedimensional plots with the axes representing average PETHs for different subpopulations. Original (C) and shuffled (D) datasets are shown.

neuron reached its peak firing rate. In the color plot showing the average PETHs (Fig. 2E), PETHs of the neurons activated early are plotted at the top and PETHs of the neurons activated late are plotted at the bottom, which results in a clear display of an activity wave running across the neurons in the population. This exact reordering was applied to the PETHs for individual conditions (Fig. 2A-D). In the PETHs for individual conditions, the temporal order of responses persisted with some jitter (e.g., compare panels A, B, C and D in Fig. 2). The same sequence of responses is also clear in the scatterplot that displays the time of peak response for different conditions and different neurons (Fig. 2F). Additionally, pairwise correlations were strong for the neurons with similar occurrences of response and weak (or negative) for the neurons with dissimilar occurrences (Fig. 2G). Thus, the PETH analysis showed that in Churchland's data neurons responded in a certain temporal order, and this order persisted to an extent when the monkey altered the way it performed a reaching movement.

To assess how the activation order of different neurons could contribute to a population rotational pattern, we split the entire neuronal population into three subpopulations: neurons with early peaks (ranks 1-73), intermediate peaks (74-146), and late peaks (147-218) (Fig. 2E). Next, we calculated average PETHs for each subpopulation and for each experimental condition (Fig. 3A). As expected, this plot revealed three groups of PETHs (early-peak, intermediate-peak and late-peak) whose shapes did not change substantially across conditions. Plotting these PETHs in a three-dimensional space (where dimensions corresponded to the subpopulations) yielded a family of curved trace (Fig. 3C) that resembled the circles obtained with jPCA (Fig. 1B). As an additional control, we randomly shuffled conditions for each neuron and plotted the curves for the shuffled data (Fig. 3B,D). Both the average PETHs for the subpopulations (Fig. 3B) and the three-dimensional plot (Fig. 3D) were little affected by the shuffling procedure, which confirmed that the activation order of the neurons was roughly the same for different conditions.

To further clarify the origin of the rotational patterns, we calculated the initial three PCs for the data of Churchland et al. and plotted them as a three-dimensional plot (Fig. 4A). The PC traces were clearly curved. 
A

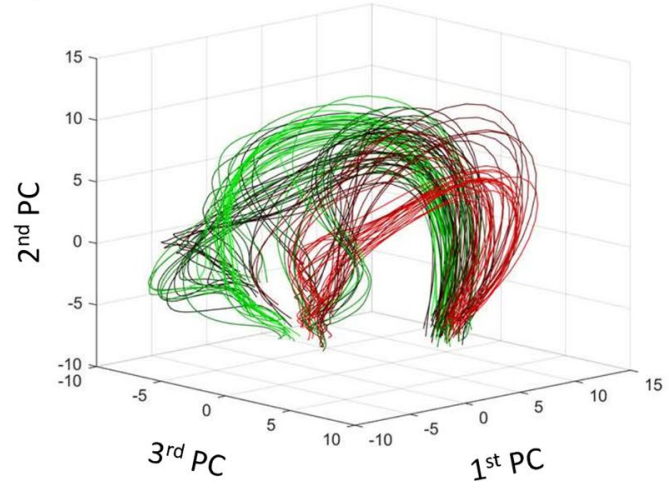

C

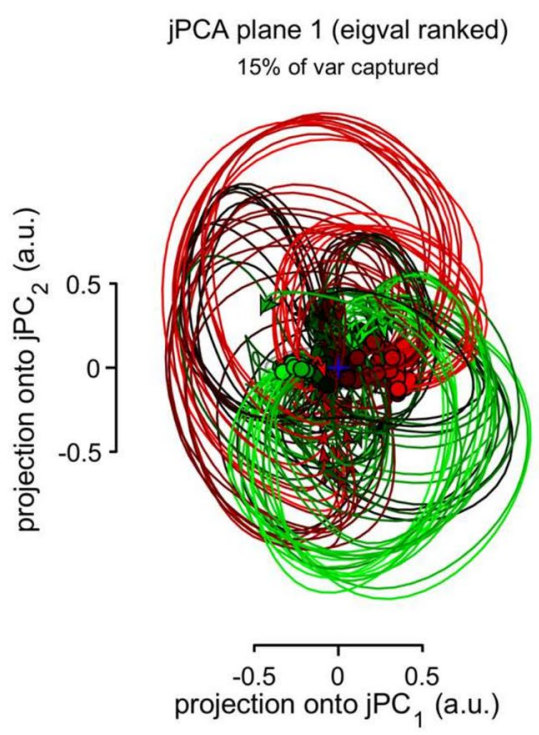

B Shuffled

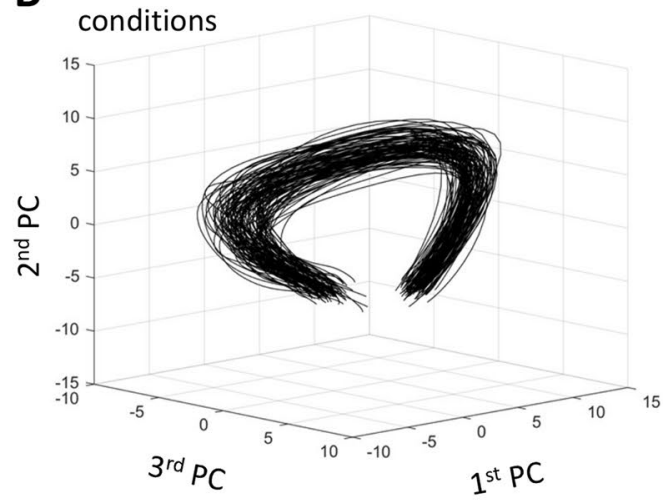

D $\begin{array}{lc}\text { Shuffled } & \text { jPCA plane } 1 \text { (eigval ranked) } \\ \text { conditions } & 4 \% \text { of var captured }\end{array}$

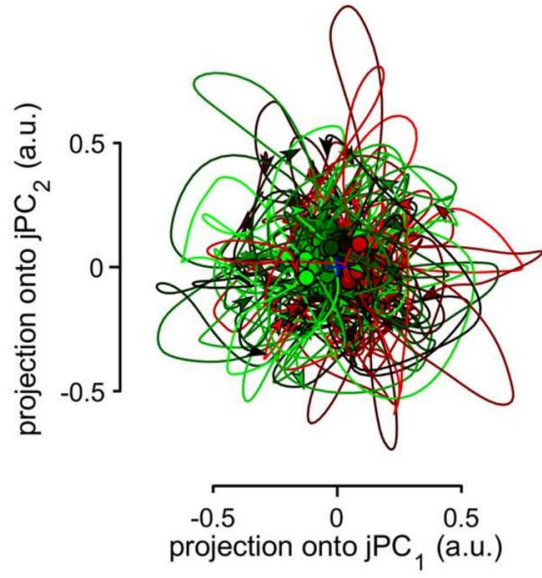

E

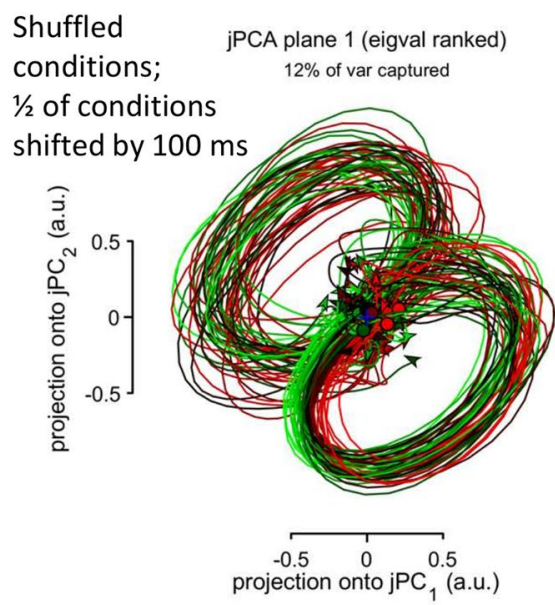

Figure 4. Rotations of principal components. (A) The first three PCs plotted as a three-dimensional plot. Color conventions as in Fig. 1B. (B) PCs for the data with shuffled conditions. (C) jPCA results for the data in (A). (D) jPCA results for the data in (B). (E) jPCA results after the shuffled data (B) was shifted forward by $100 \mathrm{~ms}$ for one-half of the conditions.

Additionally, distinct clusters of conditions were visible in the plots, each of them containing several traces that had similar shapes. The clusters started from approximately the same point but separated toward the end of the trial. Despite the differences between the clusters, they rotated in approximately the same fashion (e.g., in the plane defined by the first and second PCs). Thus, "rotational dynamics" were clearly visible even before the application of jPCA. As to JPCA, it also yielded several clusters of circles (Fig. 4C). 


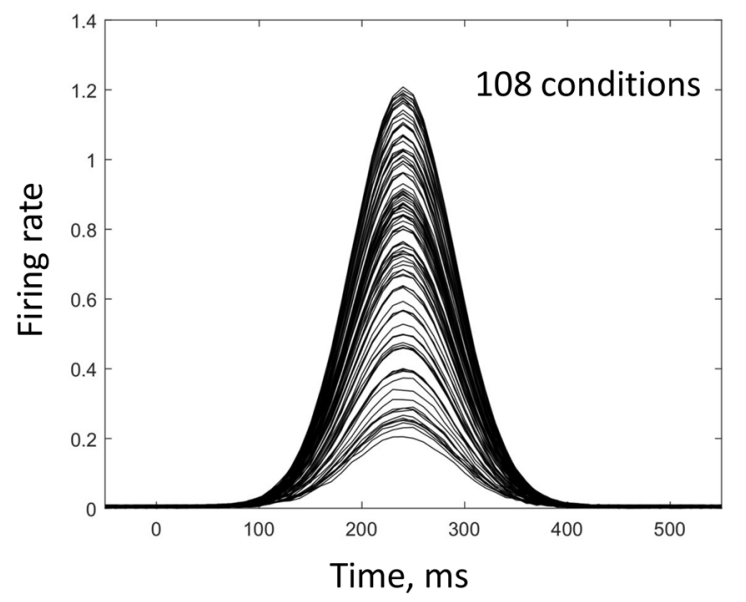

Figure 5. Simulated responses for an individual neuron. Neuronal responses were simulated using a Gaussian function. Response amplitude was randomly drawn for each condition from a uniform distribution (in the interval 0.2-1.2).

Rotations of PC traces remained even after experimental conditions were randomly shuffled for each neuron (Fig. 4B), which indicated that condition-specific correlation between the responses of different neurons was not crucial for a rotational pattern to occur. While the PC traces for the shuffled data were clearly curved, they were not clustered any more after the shuffling procedure. Unlike PCA results, jPCA failed to detect these obvious curvatures and returned noisy traces (Fig. 4D). This result suggested that, after the shuffling procedure made the population responses very similar for different conditions, the data became ill-conditioned ${ }^{16}$ for $\mathrm{jPCA}$. We, however, found a simple solution to this problem. We introduced variability to the entries for different conditions: for half of the conditions (conditions 55-108), the PETHs of all neurons were shifted forward by $100 \mathrm{~ms}$, and the initial $100 \mathrm{~ms}$ of the PETHs were assigned constant values that were equal to the first point of each original PETH. Such activity pattern would have occurred if the monkey delayed movement initiation by $100 \mathrm{~ms}$. Importantly, this manipulation did not alter the temporal sequence of neuronal population. Following the introduction of this shift, jPCA returned circles (Fig. 4E).

As a side note, jPCA with default settings encounters the same problem for a simple simulation where responses of a half of the neurons are a sine function and responses of the other half are a cosine. Although this is an obvious case of the presence of "rotational dynamics", jPCA fails to generate circles. However, the circles appear after the population responses are shifted in time by a random amount for different conditions. As explained below, the problem of ill-conditioning occurs because jPCA incorporates by default a data preprocessing step where, for each neuron, an across-condition average PETH is subtracted from all PETHs for that neuron.

Having established that neurons were activated in consistent temporal order in Churchland's data, we examined this effect further using a simple simulation of population activity. This simulation did not incorporate any features specific for motor cortical responses. For example, simulated neurons were not directionally tuned and instead each of them exhibited random amplitude of response for different conditions. Because of this randomness, response amplitude was not correlated in any pair of neurons. The only nonrandom pattern that we simulated was the presence of a temporal sequence in the responses in different neurons. The shape of simulated PETHs was a Gaussian function with an amplitude drawn from a uniform distribution (Fig. 5). We simulated 218 neurons and 108 conditions to match Churchland's data.

Recently, Michaels, et al. ${ }^{17}$ used a somewhat similar simulation of motor cortical neurons with a temporal sequence of responses that was consistent across conditions. Like our simulation, they observed an occurrence of "rotational dynamics" under these conditions. Their simulation was, however, more sophisticated than ours as they simulated directionally tuned motor cortical neurons and attempted to compare a representational model of population activity with a dynamical-system model. To prove that jPCA reveals "complex aspects" of motor cortical activity, they designed sophisticated methods for data permutations. Yet, they did not consider the possibility that some of these permutations made their simulated data ill conditioned, which resulted in jPCA failing to return circular trajectories. In our simulation, resemblance to motor cortical activity was minimal. We also applied a simple correction to cope with the ill conditioned data. Moreover, our overall conclusion is the opposite to the opinion of Michaels et al. who highly valued jPCA approach and used it as the gold standard for assessing the results of their simulation. In addition to the study of Michaels et al., other mentions can be found in neurophysiological literature of the relationship between neuronal sequences and rotations found in PC planes. Thus, Hall et al. ${ }^{18}$ noted that "rotation in the PC plane does not require underlying oscillatory sources that are orthogonal, because any consistent phase difference, or a traveling wave appearing with a different phase on each electrode, could equally be decomposed into orthogonal components", and Xu et al. ${ }^{19}$ reasoned that "state-space rotation implies neurons are consistently active at different phases of a cycle".

We started with a simulation, where the responses of different neurons were shifted in time: neuron $i$ responded $1 \mathrm{~ms}$ later than neuron $i-1$ (Fig. 6A). This simulation produced a wave of activity running through the neuronal population. Here, the population responses were very similar for different conditions as in the 
A

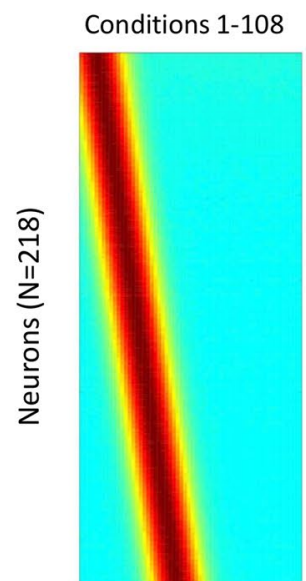

C

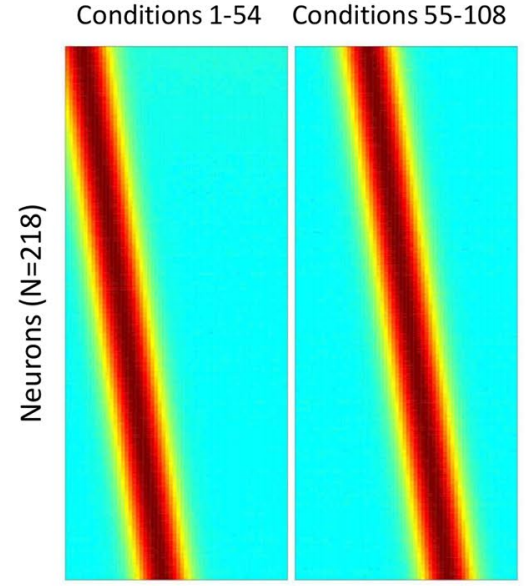

E

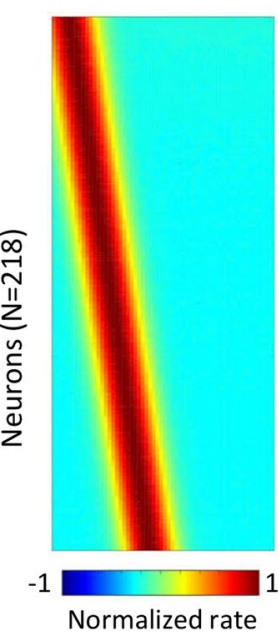

B

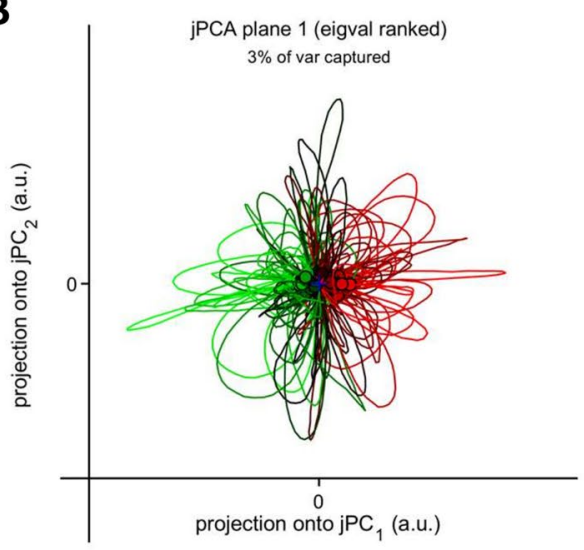

D

jPCA plane 1 (eigval ranked)
$43 \%$ of var captured

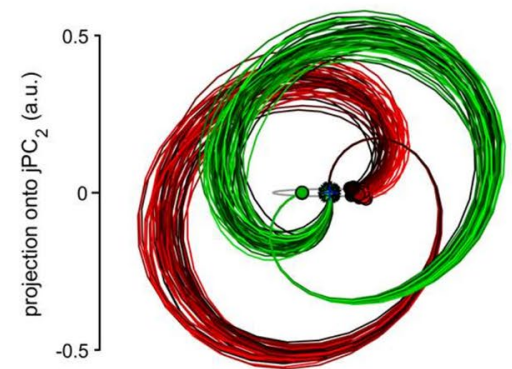

-0.5 projection onto jPC, (a.u.) 0.5

F
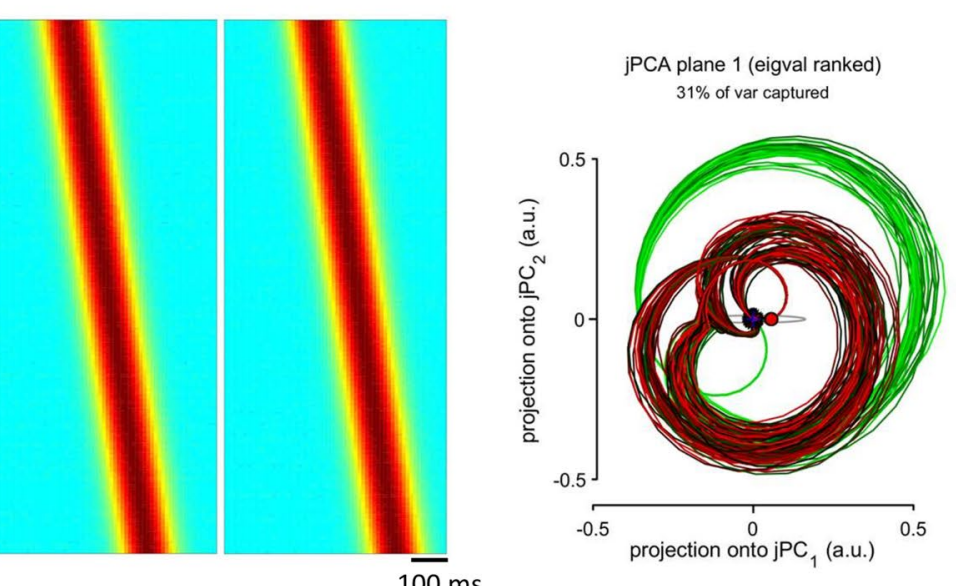

Figure 6. Simulated population responses with a time spread of peak firing rates. (A) PETHs for a simulated wave of population activity, where response of neuron $i$ occurs $1 \mathrm{~ms}$ later than response of neuron $i$-1. (B) jPCA results for the data in (A). (C) The same population wave as in A with an early onset for conditions1-54 and late onset for conditions 55-108. (D) jPCA results for the data in (C). (E) The same population wave as in $(\mathbf{A}, \mathbf{C})$ with three different onsets for conditions 1-36, 37-72 and 73-108. (F) jPCA results for the data in (E).

results for shuffled data presented above. Apparently, in this case the data was ill-conditioned for jPCA, and this is why this analysis returned noise (Fig. 6B) even though the input data contained a consistent temporal sequence of neuronal responses. To make neuronal responses more variable across conditions and thereby remove the ill conditioning, we simulated two groups of conditions: for conditions 1-54, the first neuron exhibited peak activity at time $t=50 \mathrm{~ms}$, and for conditions $55-108$ the time of its peak activity was $t=200 \mathrm{~ms}$ (Fig. 6C). The structure of the activity wave (i.e. the rule that neuron $i$ responded $1 \mathrm{~ms}$ later than neuron $i-1$ ) was the same for both groups of trials. This slight alteration of the data, which did not change the structure of the population response, was 
A

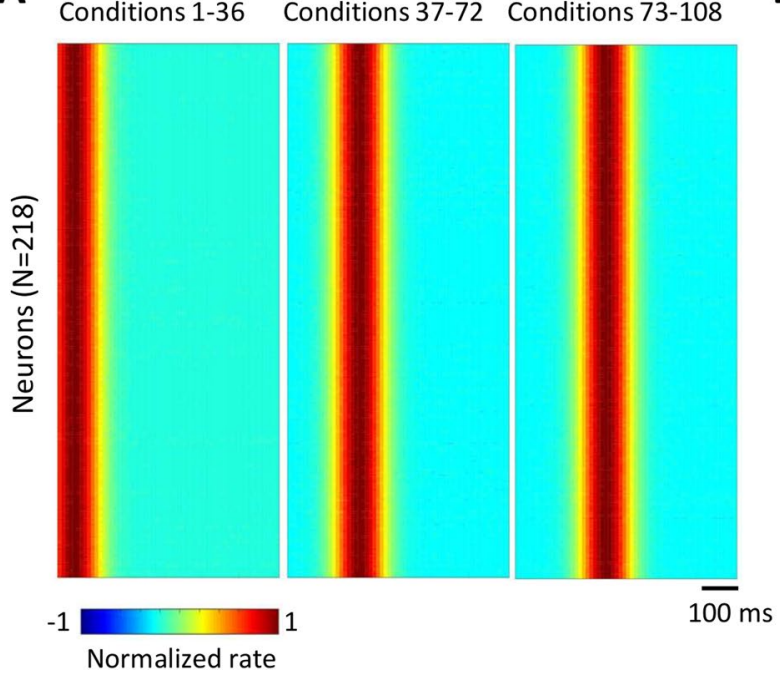

B

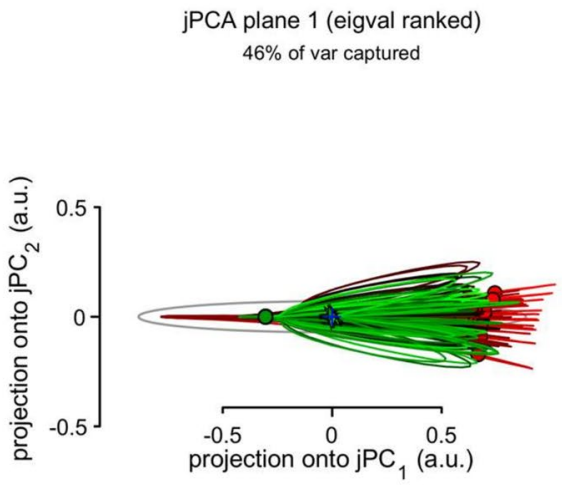

Figure 7. Simulated population responses without a time spread of peak firing rates. (A) PETHs with different response onsets for conditions 1-36,37-72 and 73-108. For each group of conditions, peak responses of different neurons were not spread in time. (B) jPCA results for the data in (A).

sufficient for jPCA to start generating circles (Fig. 6D). We also simulated three groups of conditions, where the first neuron's peak rate occurred at 50,150 and $200 \mathrm{~ms}$ for the first, second and third groups, respectively (Fig. 6E). The structure of the population wave was the same for all three groups. In this case, again, jPCA returned circles (Fig. 6F).

To verify that a temporal sequence of activation was necessary for the rotation pattern to occur for our simple model of neuronal responses, we simulated a neuronal population where all neurons responded simultaneously (Fig. 7). The shapes of neuronal responses were the same as in the previous simulation (Fig. 5). In this case, jPCA failed to generate circles (Fig. 7B) even when three groups of conditions were simulated with shifted activity onsets (Fig. 7A).

In addition to running jPCA with the default setting, where an average across-condition response was subtracted from each PETH, we ran jPCA without this setting (Fig. 8). In this case, the problem of ill conditioning did not occur, and jPCA returned circles in all cases with the exception of the simulation, where there was no temporal sequence of neuronal responses (Fig. 8D). Overall, probing our simulated data with different versions of Churchland's jPCA supported the hypothesis that a temporal sequence of neuronal responses results in a population rotation pattern, with a caveat that the problem of data ill-conditioning had to be handled when jPCA with the default settings was applied.

Finally, we probed a more direct approach for converting Churchland's data into circular trajectories. Churchland et al. linearly fit the population activity vector to its first derivative (Eq. 1) with the goal of extracting a rotational structure. We hypothesized that such an extraction of a desired "population property" could be achieved with a multiple linear regression. Accordingly, we utilized multiple linear regression to fit neuronal data to a circle:

$$
x=\cos \frac{2 \pi t}{\mathrm{~T}} ; y=\sin \frac{2 \pi \mathrm{t}}{\mathrm{T}}
$$

or to the Lissajous curve shaped as $\infty$ :

$$
x=\cos \frac{2 \pi t}{\mathrm{~T}} ; y=\sin \frac{4 \pi \mathrm{t}}{\mathrm{T}}
$$

where $t$ is time and $T$ is trial duration. The regression worked well for both shapes (Fig. 9A,B), and it even generalized to new conditions, as evident from the analysis where half of the data were used for training the regression model and the other half for generating predictions (Fig. 9C,D). The shuffled data could be fit to the Lissajous curves, as well (Fig. 9E-H), although the prediction of $\infty$ was very noisy (Fig. 9H).

\section{Discussion}

To clarify the neurophysiological significance of "rotational dynamics", we conducted additional analyses of Churchland's data and performed simple simulations. In the original dataset, we discovered a temporal order in which the neurons were activated and found that this order was relatively consistent across conditions. In our opinion, this is a useful observation that has not been described in sufficient detail in the original article of Churchland et al. and the publications that emerged from their study. We suggest that simple plots of population PETHs (e.g., Fig. 2) should be used to clarify any results on "rotational dynamics". These plots could be very useful to clarify claims like the "rotational dynamics" persist even though motor-related patterns are different across condition ${ }^{12}$ or the recurrent neural network trained to generate arm-muscle EMGs develops rotational patterns ${ }^{20}$. 


\section{A Original data}

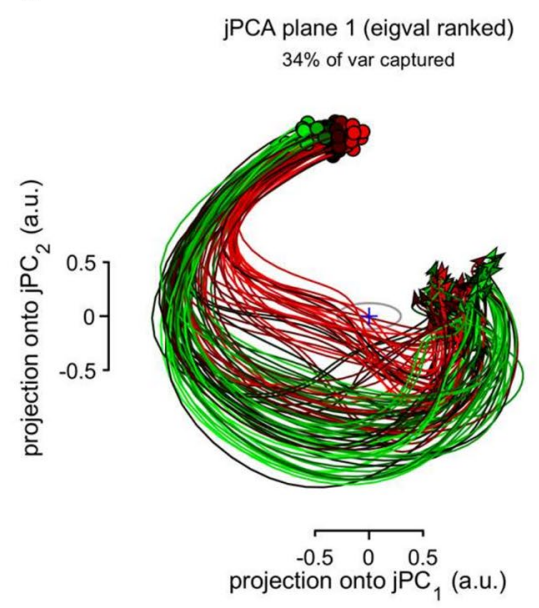

C Simulated data with a temporal sequence

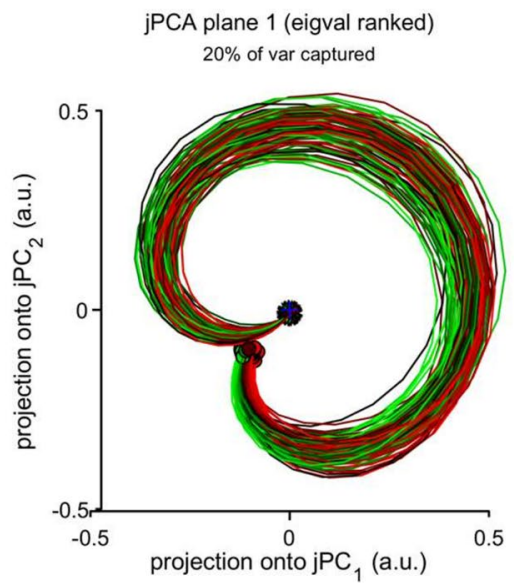

B Original data with shuffled conditions

jPCA plane 1 (eigval ranked) $19 \%$ of var captured

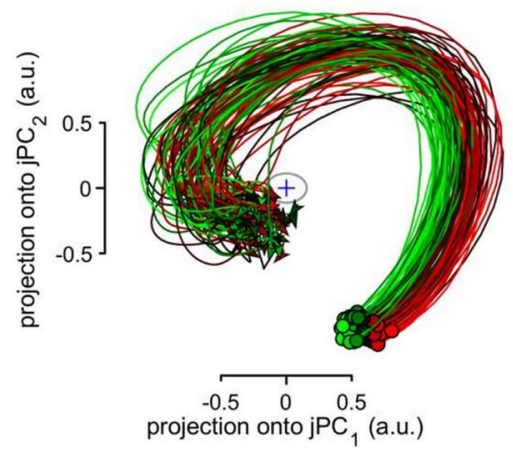

D Simulated data without a temporal sequence

jPCA plane 1 (eigval ranked) $72 \%$ of var captured
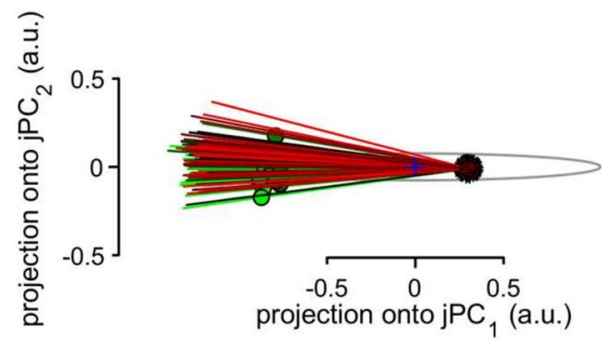

Figure 8. jPCA results without subtracting across-condition average responses. (A) Original data of Churchland et al., the same data as in Fig. 4C. (B) Original data after across-condition shuffling, the same data as in Fig. 4D. (C) Simulated data with a temporal sequence of responses, the same data as in Fig. 5A,B. (D) Simulated data without a temporal sequence of responses, the same data as in Fig. 7.

The existence of a temporal spread in neuronal peak rates (or response latencies) has been extensively documented in the literature. For example, waves of neuronal discharges similar to those shown in Fig. 2E,F have been depicted in numerous publications ${ }^{21-26}$. Sequential structure has been reported in the frequency domain, as well ${ }^{27-29}$. As to motor cortical patterns during reaching, Fig. 7 in Georgopoulos et al. ${ }^{2}$ shows a distribution of the times of onset of the first increase in discharge in motor cortical neurons for center-out arm reaching movements performed by a monkey. The onsets were calculated for the neurons' preferred directions and ranged -300 to $500 \mathrm{~ms}$ relative to movement initiation time. In a more recent paper ${ }^{30}$, we have demonstrated such a spread for simultaneously recorded populations of neurons recorded in the motor and primary somatosensory cortical areas.

It may be true that no previous paper focused on the consistency of neuronal activation order for a range of movements. Yet, Churchland and his colleagues did not emphasize such consistency either and instead emphasized variability of individual neuronal responses across conditions. ("Responses were typically complex, multiphasic and heterogeneous"). According to their explanation, rotational patterns are principally a population phenomenon that persists despite individual neurons exhibiting different activity patterns for different arm reaches. The same interpretation of Churchland's results can be found in more recent literature ${ }^{17,31,32}$. For example, according to Kaufman et al. ${ }^{32}$, "while individual neurons may exhibit confusing responses, the population-level, dynamical systems view may provide insight about how a circuit performs its key computations". In other words, according to Churchland et al. and their followers, dimensionality reduction technique is needed for making sense of how neurons as a population modulate their activity as time passes. 
A

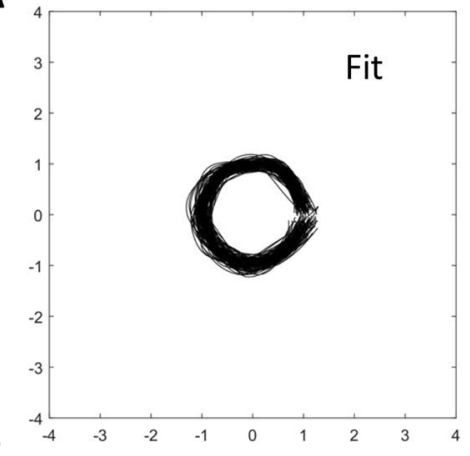

C

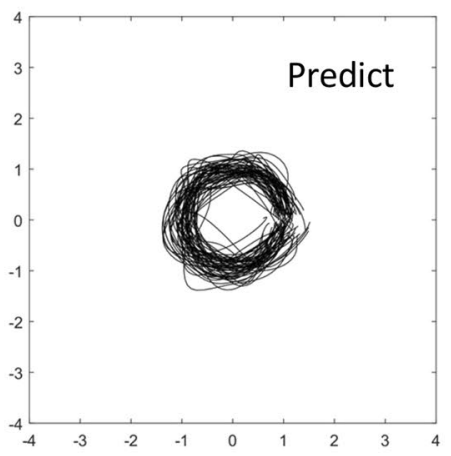

B

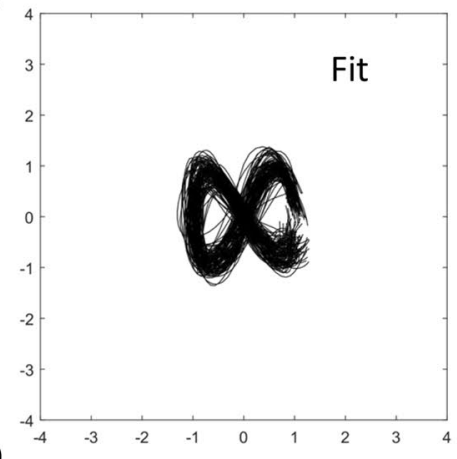

D

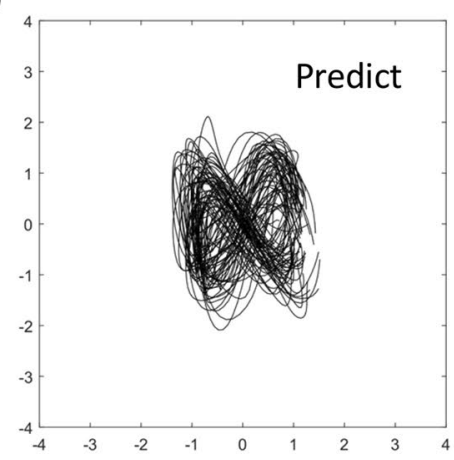

Shuffled conditions

E

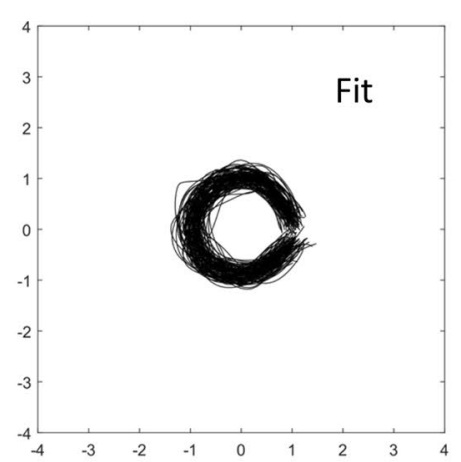

G

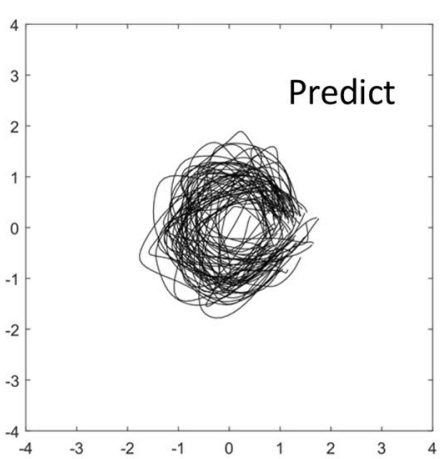

$\mathbf{F}$

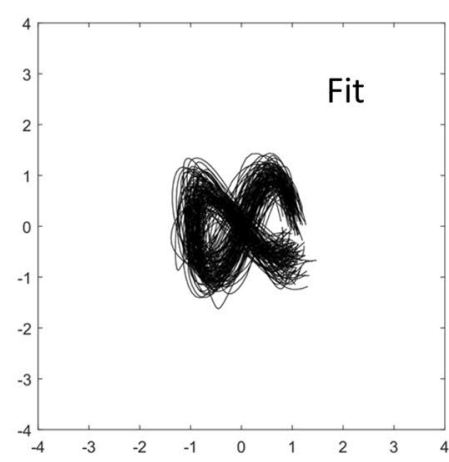

H

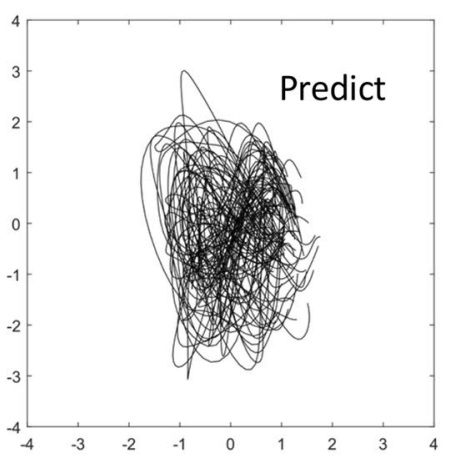

Figure 9. Fitting Churchland's neuronal population data to Lissajous curves. (A) Fitting to a circle. (B) Fitting to the curve shaped as $\infty$. (C) Prediction of a circle. Half of the conditions were used to train the regression model; the other half to generate prediction. (D) Prediction of the $\infty$ shape. (E-H) Fitting and predicting for the data with shuffled conditions.

Temporal sequences of neuronal responses that are consistent across a set of experimental conditions could occur for various reasons. For example, such a temporal order could be related to serial information processing by a neuronal circuit. Orderly response onsets have been reported for the activity of multiple cortical areas transforming sensory inputs into behavioral actions. For example, de Lafuente and Romo ${ }^{33}$ reported that a wave of neuronal activity travelled from the somatosensory cortex to the premotor cortical areas when monkeys performed a task that required perceptual judgment of a tactile stimulus. Sensorimotor transformation of this kind 
could be analyzed using a comparison of response characteristics for different cortical locations and different types of neurons. Unfortunately, jPCA makes such an analysis impossible because it lumps the activity of many neurons together (e.g. compare Fig. 2 with Fig. 4C).

Based on the results of our analysis, we question that Churchland's results provide a strong evidence in favor of the dynamical-system that acts "like a spring box" that "could be released to act as an engine of movement" (an allegory used by Michaels, et al. ${ }^{17}$ ). This is simply because the mere presence of a temporal sequence of neuronal responses tells us very little about the properties of the system even if we assume that it is dynamical. Temporal lags between the responses of different neurons could occur for various reasons, including direct connectivity, common input, or association with different stages of information processing. jPCA would reveal rotations even in the combined data from different monkeys - an obviously artifactual cause for lags between the responses of different neurons, and there would not be a way to tell that this is not a "dynamical system". (Unless correlations between the responses of different neurons are analyzed across behavioral trials; but such an analysis is not incorporated in the Churchland et al. approach).

The finding that neurons in some parts of the nervous system respond to a stimulus with different delays (and jPCA reveals rotations) is not surprising as it has been published in myriads of papers. For example, in sensory systems, peripheral receptors are activated first, followed by neuronal activity in the spinal cord, brainstem, thalamus and eventually cortex. Although this sensory processing chain in principle could be defined as a dynamical system, this definition alone does not illuminate any mechanism of sensory processing. By the same token, motor systems of the brain are well-known to perform sensorimotor transformations ${ }^{34,35}$. In Churchland's case, this is the transformation of a go-cue and preceding instructions into a motor execution command. Saying that "rotational dynamics" occur during this sensorimotor transformation only adds terminology but does not clarify any concrete neuronal mechanism.

According to Churchland et al., "a focus on the dynamics that generate movement will help transcend the controversy over what single neurons in motor cortex code or represent". While we agree with this general idea, we do not see how their jPCA results transcend any controversy, particularly given the fact that population rotations could be obtained with little effort using simple and quite arbitrary simulations that did not mimic any important property of motor cortical activity. Without a linkage to a specific function of the motor cortex, jPCA appears to be just a way to produce an illustration of the lags between the responses of different neurons.

Although analysis of response lags in different neurons could be valuable for understanding neural processing pipelines, we are not convinced that jPCA is the best tool for such an analysis. We observed cases where temporal sequences of responses were revealed with several methods but not with jPCA set to default, where for each neuron an across-condition response was subtracted from this neuron's PETHs. Thus, shuffling Churchland's data across conditions did not destroy the rotations revealed with non-jPCA approaches (Figs. 3B,D and 4B) but jPCA failed to produce circular trajectories in this case (Fig. 4D). This happened because jPCA was designed to reveal "those aspects of the neural response that differ across conditions", to avoid "more trivial possibilities", and to "interpret projections where activity unfolds differently across conditions". These goals that are not entirely clear to us were achieved by calculating an across-condition average PETH for each neuron and then subtracting this average response from all PETHs for that neuron. When we removed this data preprocessing step from jPCA, jPCA generated circles for the shuffled data (Fig. 8B) and simulated temporal sequences of neuronal responses (Fig. 8C). Looking closer at the preprocessing procedure employed by Churchland et al., we find it arbitrary and questionable. The first concern is that this data manipulation violates the assumptions of Eq. 1, where true neuronal rates are used instead of neuronal rates corrected by subtracting an average contribution from different conditions. If Churchland et al. wanted to hypothesize that an average response influenced population dynamics, they should have incorporated this function of time in Eq. 1 and explained what brain structure could generate it. Unless such an explanation is provided, it is unclear why one would need to subtract an across-condition average from the neuronal response. Consider an example of a mass on a spring, a harmonic oscillator that could be described as a dynamical system ${ }^{36}$. It would make little sense to analyze the properties of this system by subtracting average responses for different conditions like different values of mass and spring constant, which influence the period of oscillations. (This example is relevant to motor control where biomechanical parameters are different for different movements). Calculation of an across-condition average is an artificial procedure because it requires aligning neuronal data on an event of interest, for example go-cue onset, movement onset, or Churchland's "neuronal movement onset". For data points distant from this aligning event, variability in neuronal responses would grow ${ }^{37}$, making the average PETH a less reliable description of neuronal activity as a function of time and, consequently, an ineffective factor for Churchland's data correction. Additionally, this correction cannot be applied to continuous behaviors like target pursuit task ${ }^{38}$, where phase differences between the activity of different neurons are very likely to occur but the task is not composed of epochs corresponding to a discrete set of conditions. Churchland and his colleagues justified their correction procedure by the desire to remove "rotations that are similar for all conditions" because they prevent one "to gain multiple views of the underlying process, making it difficult to infer whether rotations are due to dynamics or to more trivial possibilities". While they did not clarify the difference between the "dynamics" and "more trivial possibilities", the consequences of the implemented data manipulation are not trivial. It may have caused aliasing, such as significant distortion of neuronal responses. Overall, this manipulation appears poorly justified and its benefits are unclear.

Mathematically, Churchland's method to produce a rotating pattern from neuronal PETHs can be described as a linear transformation with a certain number of free parameters. With a sufficient number of free parameters, multiple linear regression can yield practically any desired curve ${ }^{39}$. For example, when we applied a multiple linear regression to Churchland's data, we easily produced a variety of Lissajous curves. Because of the similarity of neuronal responses across conditions, this transformation even generalized from the training dataset (half of the conditions) to new data in the test dataset (the other half) (Fig. 8). Evidently, no one would claim that any of 
these arbitrarily chosen curves represents a physiologically meaningful neural population mechanism, although we used a linear transformation very similar to the one Churchland and his colleagues employed to generate their curves.

Altogether, our results challenge the conclusions of Churchland and his colleagues. Clearly, their effects simply reflect what one should expect by applying a linear transformation, such as PCA, to reduce the dimensionality of large-dimension data and then adjusting the resulting components to a new basis that reproduces a desired behavior (in this case rotation). $\mathrm{PCA}^{10}$ and independent component analysis ${ }^{11}$ were introduced twenty years ago by our group to extract correlated neuronal patterns and reduce dimensionality of neuronal-ensemble data (recently reviewed ${ }^{31,40}$ ). While the findings of Churchland and his colleagues can be viewed as an extension of this approach and a method to produce a visually appealing phase plot, we doubt that they have discovered any new neurophysiological mechanism, as claimed in their article. Indeed, the mere fact that neurons in a population respond at different times with respect to a go-cue tells very little about the function of these responses and does not discard any of the previously proposed representational interpretations, such as the suggestion that neuronal populations perform a sensorimotor transformation ${ }^{34,35,41}$, handle specific motor parameters ${ }^{2-5}$, and/or respond to movement-related sensory inputs ${ }^{42-45}$.

Cortical activity is dynamical in the sense that neuronal rates change in time. Differential equations ${ }^{46}$ and other mathematical methods $s^{13,47-49}$ are certainly applicable to modeling these dynamics. However, a much more thorough analysis compared to the method proposed by Churchland et al. is needed for making a major advance in our understanding of cortical mechanisms of motor control. In light of the exponential increase in simultaneous neural recording ${ }^{7,50}$ and also the future impact of new technologies such as NeuroPixels ${ }^{51}$, it is therefore perhaps an appropriate moment to emphasize that pitfalls may await if we rely too heavily on low-dimensional representations of population activity, such as jPCA.

\section{Methods}

The data (single units and good multiunits recorded in monkey N; the data used to construct Fig. 3 f of Churchland et al.) and MATLAB scripts were obtained from the Churchland lab's online depository (https://www.dropbox.

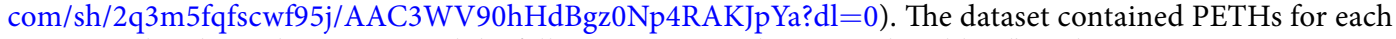
neuron and each condition. We used the following commands to run Churchland's code:

$$
\begin{gathered}
\text { times }=-50: 10: 550 ; \\
j P C A \_ \text {params. numPCs }=6 ; \\
{[\text { Projection, Summary }]=j P C A(\text { Data }, \text { times, jPCA_params }) ;}
\end{gathered}
$$

This corresponds to the time range -50 to $550 \mathrm{~ms}$ and six PCs entered in jPCA.

To produce the plots shown in Fig. 2A-D, we stacked Churchland's PETHs together, and Fig. 2E shows PETHs averaged across conditions. The average PETHs were used to find peak firing rates and the time of their occurrences. PETHs of Fig. 2A-E were sorted according to the sequence of these peaks of the average PETHs. To improve the display of phase shifts between the neurons, PETHs of Fig. 2A-E were normalized by subtracting the mean and dividing by the peak PETH value. This normalization was used only for plotting the graphs of Fig. 2A-E but not for calculating average PETHs for individual neurons (Fig. 2E) or neuronal subpopulations (Fig. 3).

In the PCA analysis (Fig. 4), we standardized PETHs for each neuron by subtracting the overall mean (i.e., average for all PETHs appended to each other) and dividing by the overall standard deviation (again, for all PETHs appended).

Simulated PETHs (Fig. 5) were computed in MATLAB as:

$$
P E T H=\exp (-(\text { times }- \text { tau }) \wedge 2 / 50) *(0.2+\operatorname{rand}(1)) ;
$$

where the time shift, tau, was selected to produce $10-\mathrm{ms}$ increments of the delay for the neurons in the sequence (Fig. 6). Neither the width of the response not the amplitude (uniformly distributed from 0.2 to 1.2 in Eq. 5) were critical for the rotations to occur. However, to cope with the ill-conditioning of the population responses highly correlated across conditions, it was important to introduce temporal variability to the simulated PETHs. This was done by offsetting tau for all neurons by the same amount of time for several groups of conditions (Fig. 6C,D).

Multiple linear regressions (Fig. 9) were implemented in MATLAB (regress function). Here, neuronal activity was transformed into Lissajous curves. Fitting (Fig. 9A,B,E,F) was conducted by using the same conditions as the training and test data. Predictions (Fig. 9C,D,G,H) were computed by using half of the trials to train the regression model and the other half to test.

\section{Data availability}

Data and scripts are available at https://www.dropbox.com/sh/svex4f947uxo53x/AABkOnIS_ertXcp1xZ-U6gO$\mathrm{a} ? \mathrm{dl}=0$.

Received: 17 May 2019; Accepted: 14 November 2019;

Published online: 12 December 2019 


\section{References}

1. Evarts, E. V. Activity of motor cortex neurons in association with learned movement. Int J Neurosci 3, 113-124 (1972).

2. Georgopoulos, A. P., Kalaska, J. F., Caminiti, R. \& Massey, J. T. On the relations between the direction of two-dimensional arm movements and cell discharge in primate motor cortex. J Neurosci 2, 1527-1537 (1982).

3. Kakei, S., Hoffman, D. S. \& Strick, P. L. Muscle and movement representations in the primary motor cortex. Science 285, 2136-2139 (1999).

4. Georgopoulos, A. P., Ashe, J., Smyrnis, N. \& Taira, M. The motor cortex and the coding of force. Science 256, 1692-1695 (1992).

5. Zhuang, K. Z., Lebedev, M. A. \& Nicolelis, M. A. Joint cross-correlation analysis reveals complex, time-dependent functional relationship between cortical neurons and arm electromyograms. J Neurophysiol 112, 2865-2887, https://doi.org/10.1152/ jn.00031.2013 (2014).

6. Nicolelis, M. A. et al. Chronic, multisite, multielectrode recordings in macaque monkeys. Proc Natl Acad Sci USA 100, 11041-11046 (2003).

7. Schwarz, D. A. et al. Chronic, wireless recordings of large-scale brain activity in freely moving rhesus monkeys. Nat Methods $\mathbf{1 1}, 670$ (2014).

8. Averbeck, B. B. \& Lee, D. Coding and transmission of information by neural ensembles. Trends Neurosci 27, 225-230 (2004).

9. Nicolelis, M. A. \& Lebedev, M. A. Principles of neural ensemble physiology underlying the operation of brain-machine interfaces. Nat Rev Neurosci 10, 530 (2009).

10. Chapin, J. K. \& Nicolelis, M. A. Principal component analysis of neuronal ensemble activity reveals multidimensional somatosensory representations. J Neurosci Methods 94, 121-140 (1999).

11. Laubach, M., Shuler, M. \& Nicolelis, M. A. Independent component analyses for quantifying neuronal ensemble interactions. $J$ Neurosci Methods 94, 141-154 (1999).

12. Churchland, M. M. et al. Neural population dynamics during reaching. Nature 487, 51-56, https://doi.org/10.1038/nature11129 (2012).

13. Pandarinath, C. et al. Inferring single-trial neural population dynamics using sequential auto-encoders. Nat Methods 15, $805-815$ (2018).

14. Garfinkel, A., Shevtsov, J. \& Guo, Y. Modeling life: the mathematics of biological systems. (Springer, 2017).

15. Lara, A. H., Cunningham, J. P. \& Churchland, M. M. Different population dynamics in the supplementary motor area and motor cortex during reaching. Nat Commun 9, 2754 (2018).

16. Golub, G. H. \& Van Loan, C. F. Matrix computations. Vol. 3 (JHU press, (2012).

17. Michaels, J. A., Dann, B. \& Scherberger, H. Neural population dynamics during reaching are better explained by a dynamical system than representational tuning. PLOS Comput Biol 12, e1005175 (2016).

18. Hall, T. M., de Carvalho, F. \& Jackson, A. A common structure underlies low-frequency cortical dynamics in movement, sleep, and sedation. Neuron 83, 1185-1199 (2014).

19. Xu, W., de Carvalho, F. \& Jackson, A. Sequential neural activity in primary motor cortex during sleep. J Neurosci 39, 3698-3712 (2019).

20. Sussillo, D., Churchland, M. M., Kaufman, M. T. \& Shenoy, K. V. A neural network that finds a naturalistic solution for the production of muscle activity. Nat Neurosci 18, 1025 (2015).

21. Peyrache, A., Benchenane, K., Khamassi, M., Wiener, S. \& Battaglia, F. Sequential reinstatement of neocortical activity during slow oscillations depends on cells' global activity. Front Syst Neurosci 3, https://doi.org/10.3389/neuro.06.018.2009 (2010).

22. Luczak, A., Barthó, P., Marguet, S. L., Buzsáki, G. \& Harris, K. D. Sequential structure of neocortical spontaneous activity in vivo. Proc Natl Acad Sci USA 104, 347-352 (2007).

23. Bulkin, D. A., Law, L. M. \& Smith, D. M. Placing memories in context: Hippocampal representations promote retrieval of appropriate memories. Hippocampus 26, 958-971 (2016).

24. Kvitsiani, D. et al. Distinct behavioural and network correlates of two interneuron types in prefrontal cortex. Nature 498, 363 (2013).

25. Gage, G. J., Stoetzner, C. R., Wiltschko, A. B. \& Berke, J. D. Selective activation of striatal fast-spiking interneurons during choice execution. Neuron 67, 466-479 (2010).

26. Ermentrout, G. B. \& Kleinfeld, D. Traveling electrical waves in cortex: insights from phase dynamics and speculation on a computational role. Neuron 29, 33-44 (2001).

27. Prechtl, J., Cohen, L., Pesaran, B., Mitra, P. \& Kleinfeld, D. Visual stimuli induce waves of electrical activity in turtle cortex. Proc Natl Acad Sci USA 94, 7621-7626 (1997).

28. Wang, X.-J. Neurophysiological and computational principles of cortical rhythms in cognition. Physiol Rev 90, 1195-1268 (2010).

29. Rubino, D., Robbins, K. A. \& Hatsopoulos, N. G. Propagating waves mediate information transfer in the motor cortex. Nat Neurosci 9, 1549 (2006).

30. Ifft, P., Lebedev, M. \& Nicolelis, M. A. Cortical correlates of Fitts' law. Front Integr Neurosci 5, 85 (2011).

31. Cunningham, J. P. \& Byron, M. Y. Dimensionality reduction for large-scale neural recordings. Nat Neurosci 17, 1500 (2014).

32. Kaufman, M. T., Churchland, M. M., Ryu, S. I. \& Shenoy, K. V. Cortical activity in the null space: permitting preparation without movement. Nat Neurosci 17, 440 (2014).

33. de Lafuente, V. \& Romo, R. Neural correlate of subjective sensory experience gradually builds up across cortical areas. Proc Natl Acad Sci USA 103, 14266-14271 (2006).

34. Kalaska, J. Reaching movements to visual targets: neuronal representations of sensori-motor transformations. Seminars in Neuroscience 3, 67-80 (1991).

35. Georgopoulos, A. P., Lurito, J. T., Petrides, M., Schwartz, A. B. \& Massey, J. T. Mental rotation of the neuronal population vector. Science 243, 234-236 (1989).

36. Hirsch, M. W., Smale, S. \& Devaney, R. L. Differential equations, dynamical systems, and an introduction to chaos. (Academic Press, (2012).

37. Renoult, L., Roux, S. \& Riehle, A. Time is a rubberband: neuronal activity in monkey motor cortex in relation to time estimation. Eur J Neurosci 23, 3098-3108 (2006).

38. Li, Z. et al. Unscented Kalman filter for brain-machine interfaces. PLOS One 4, e6243 (2009).

39. Babyak, M. A. What you see may not be what you get: a brief, nontechnical introduction to overfitting in regression-type models. Psychosom Med 66, 411-421 (2004).

40. Paninski, L. \& Cunningham, J. P. Neural data science: accelerating the experiment-analysis-theory cycle in large-scale neuroscience. Curr Opin Neurobiol 50, 232-241 (2018)

41. Kakei, S., Hoffman, D. S. \& Strick, P. L. Sensorimotor transformations in cortical motor areas. Neurosci Res 46, 1-10 (2003).

42. Bötzel, K., Ecker, C. \& Schulze, S. Topography and dipole analysis of reafferent electrical brain activity following the Bereitschaftspotential. Exp Brain Res 114, 352-361 (1997).

43. Alary, F. et al. Event-related potentials elicited by passive movements in humans: characterization, source analysis, and comparison to fMRI. Neuroimage 8, 377-390 (1998).

44. Goldring, S. \& Ratcheson, R. Human motor cortex: sensory input data from single neuron recordings. Science 175, 1493-1495 (1972).

45. Rosen, I. \& Asanuma, H. Peripheral afferent inputs to the forelimb area of the monkey motor cortex: input-output relations. Exp Brain Res 14, 257-273 (1972). 
46. Lukashin, A. V. \& Georgopoulos, A. P. A dynamical neural network model for motor cortical activity during movement: population coding of movement trajectories. Biol Cybern 69, 517-524 (1993).

47. Moody, S. L. \& Zipser, D. A model of reaching dynamics in primary motor cortex. J Cogn Neurosci 10, 35-45 (1998).

48. Kawato, M. \& Wolpert, D. Internal models for motor control. Sensory guidance of movement 218, 291-307 (1998).

49. Todorov, E. Direct cortical control of muscle activation in voluntary arm movements: a model. Nat Neurosci 3, 391 (2000).

50. Stevenson, I. H. \& Kording, K. P. How advances in neural recording affect data analysis. Nat Neurosci 14, 139 (2011).

51. Steinmetz, N. A., Koch, C., Harris, K. D. \& Carandini, M. Challenges and opportunities for large-scale electrophysiology with Neuropixels probes. Curr Opin Neurobiol 50, 92-100 (2018).

\section{Acknowledgements}

This research was supported by the NIH Director's Pioneer award (DP1-OD006798) and by NIH's National Institute of Neurological Disorders and Stroke award (R01NS073952) to M.A.L.N. The content is solely the responsibility of the authors and does not necessarily represent the official views of the Office of the NIH Director or the NIH. Alexei Ossadtchi was partially supported by the Center for Bioelectric Interfaces NRU HSE, RF Government grant, ag. No. 14.641.31.0003.

\section{Author contributions}

M.A.L. and M.A.L.N. wrote the paper. M.A.L. analyzed the data. A.O., N.A.M., N.A.U. and M.R.C. assisted in data analysis. All authors discussed the arguments provided in the paper.

\section{Competing interests}

The authors declare no competing interests.

\section{Additional information}

Correspondence and requests for materials should be addressed to M.A.L.N.

Reprints and permissions information is available at www.nature.com/reprints.

Publisher's note Springer Nature remains neutral with regard to jurisdictional claims in published maps and institutional affiliations.

(c) (i) Open Access This article is licensed under a Creative Commons Attribution 4.0 International License, which permits use, sharing, adaptation, distribution and reproduction in any medium or format, as long as you give appropriate credit to the original author(s) and the source, provide a link to the Creative Commons license, and indicate if changes were made. The images or other third party material in this article are included in the article's Creative Commons license, unless indicated otherwise in a credit line to the material. If material is not included in the article's Creative Commons license and your intended use is not permitted by statutory regulation or exceeds the permitted use, you will need to obtain permission directly from the copyright holder. To view a copy of this license, visit http://creativecommons.org/licenses/by/4.0/.

(C) The Author(s) 2019 